\title{
A LOG-EXP Still Image Compression Chip Design ${ }^{*}$
}

\author{
Sheng-Chieh Huang and Liang-Gee Chen
}

\author{
Department of Electrical Engineering, National Taiwan University, \\ Taipei, Taiwan, R.O.C.
}

\begin{abstract}
In this paper, a fully pipelined single chip is proposed for the $L O G-E X P$ still image compression. The design of the LOG-EXP image compression focus on the high compression ratio of the complex texture (e,g. benchmark image baboon) and high quality image, especially the PSNR requirement above 36 . In comparison with the JPEG compression result (bpp $=0.99$, PSNR = 26.9), this compression algorithm uses less bpp (bpp = 0.87 ) to get higher image quality ( $P S N R=36.38$ ) for the benchmark image baboon. The entire LOG-EXP image compression system can be implemented on a single chip to yield a clock rate of $175 \mathrm{MHz}$ which allow an input rate of 30 frames per second for $1024 \times 1024$ color images.
\end{abstract}

\section{Introduction}

In recent years, the networking multimedia systems are popular in life. For example, the WWW, video conference, video phone, DVD, Set-Up Box, HDTV, Digital TV, Digital Still Camera (DSC) are the trend of the consumer electronic applications. The high image compression ratio, high quality and high speed are the basic elements of the efficient image transmission system. So, the JPEG still image compression standard is widely used in many multimedia applications.

But the block artifact of the JPEG destroys the image quality seriously, especially in the zoom view of the image. Besides, the new requirements in the large size and true color resolution image, the JPEG can not afford the needs of the high throughput. In this paper, a novel VLSI architecture based on LOG-EXP compression is proposed to satisfy the high speed requirement and avoid the block artifact of the high quality image compression.

\section{Algorithm Design}

Based on the logarithmic number system (LNS) properties, the LOG-EXP still image compression system is designed [1]. The compression and decompression flow is shown on Fig. 1. For the gray level image, the pixel value is usually represented in integer format. In the LNS format, the large neighboring difference between the two pixels can be reduced into small range. Due to the finite word length effect and the continuous distribution of the pixel value, the original 8 bits gray level image can be represented in 7 bits LNS format.

To compute the differences between the neighbor pixels, the neighboring differences will be very small and there are many same differences. For removing the large neighboring difference from this line to the next line, the snake scan is used.

The repeat reduction of the neighboring difference is shown in Fig. 2. After reducing the repeat values, the data part and times part are processed with the Huffman encoder to remove the redundancy among the large amount of similar and regular neighboring differences.

\section{Architecture Design and Implementation}

The LOG-EXP encoder and decoder consist of four parts. These parts are divided into the different pipeline stage and shown in Fig. 3. and Fig.4. Each part is described in the following 3.1 Logarithmic and Exponential Transform

The logarithmic/exponential function table lookup is used to compute the logarithmic and exponential transform. The range of the pixel value is from 0 to 255 . So, the LOG/EXP table is 8 bits input and 8 bit logarithmic number output. Besides, this table is also used to transform the coded pixel to the original pixel with exponential function.

3.2 Difference Calculation and Complement

The adder/subtractor calculates the pixel difference for encoding and adds the difference to the previous pixel value for decoding. Because the 8 bit pixel values are transformed into the 7 bit logarithmic format, the adder/subtractor computes in 7 bits input/output.

\subsection{Repeat Item Reduction and Restore}

In the encoder phase, the comparator compares the present data with the previous one for finding the repeat items. If the comparison result is true, the times counter $(+1$ counter) records the repeat times and disables the Huffman encoder operation. Until the comparison result becomes false, the Huffman encoder is enabled and the pixel difference and times data in the Huffman buffer are used as index for Huffman table lookup.

At the part of the repeat item restore, the times data gets from the Huffman buffer and setup the times counter ( -1 counter) to decrease the repeat times. When the times counter become zero, the Huffman decoder is enabled to process the new data. If the times are still not to be zero, the multiplexer chooses the previous same value as the pixel difference for the decoding phase.

\subsection{Huffman Encoder and Decoder}

The Huffman table is addressed with the 15 bits index (the 7 bits difference value and the 8 bits times) and the output is the 8 bits format for the storage of the coded bitstream register.

According the previous implementation results [2][3], the 8 bit LOG/EXP table lookup can be implemented in the 5 ns delay and the critical path delay of the Huffman encoder/decoder is $5.72 \mathrm{~ns}$ in PLA lookup table. The difference calculation and complement part consists of the 7 bit adder and the 2's complement circuit. With the Synopsys verilog simulation using the $0.6 \mu \mathrm{m}$ COMPASS cell library, the critical path delay is 5.03 $\mathrm{ns}$. The speed of the comparator and times counter part is $1.27 \mathrm{~ns}$. From the above pipeline stage analysis, the critical path delay of

"This work was supported by National Science Council under Grant NSC87-2215-E-002-028. 
the LOG-EXP CODEC architecture is dominated by the Huffman encoder/decoder and the chip speed can achieve 175 $\mathrm{MHz}(5.72 \mathrm{~ns})$.

\section{Evaluations}

The comparison is shown in the following table between the LOG-EXP and five JPEG architectures.

\begin{tabular}{|c|c|c|}
\hline & Process (CMOS) & Speed (MHz) \\
\hline$[4]$ & $1.2 \mu \mathrm{m}$ & 20 \\
\hline$[5]$ & $1.2 \mu \mathrm{m}$ & 16.67 \\
\hline$[6]$ & $1.0 \mu \mathrm{m}$ & 30 \\
\hline$[7]$ & $1.0 \mu \mathrm{m}$ & 100 \\
\hline Proposed & $0.6 \mu \mathrm{m}$ & 175 \\
\hline
\end{tabular}

Table. 1. VLSI Architectures Comparison.

The high quality image needs high PSNR. The following table shows the comparison for the benchmark baboon:

\begin{tabular}{|c|c|c|}
\hline Log-Exp PSNR & 41.31 & 36.38 \\
\hline Log-Exp Compression Ratio & 7.34 & 9.19 \\
\hline JPEG PSNR & 37.23 & 34.53 \\
\hline JPEG Compression Ratio & 2.39 & 2.98 \\
\hline
\end{tabular}

Table. 2. The Compression when PSNR above 36.

The Fig. 5. shows the benchmark image baboon's left eye part. Though the bit per pixel (bpp) of the JPEG compression is more than the Log-Exp compression, the image quality of the Log-Exp is superior to the JPEG. Besides, the block artifact of the JPEG image is very obvious in this figure. The Log-Exp compression is processing in pixel-by-pixel, there is no block artifact drawback.

\section{References}

[1]. S. C. Huang, L.G. Chen, "LOG-EXP Compression System Design and Implementation," IEEE International Sym. on Consumer Electronics, 1998.

[2]. S.C. Huang, L.G. Chen, T.H. Chen, "A 32-bit Logarithmic Number System Processor," Journal of VLSI Signal Processing Systems for Signal, Image, and Video Technology, Vol. 14, No. 3, pp. 311-319, Dec. 1996.

[3]. H. C. Chang, L. G. Chen, Y. C. Chang, S. C. Huang, "A VLSI Architecture Design of VLC Encoder for High Data Rate Video/Image Coding," is accepted by IEEE Sym. on Circuit and System, 1999.

[4]. M. Bolton, R. Boulton, J. Martin, S. Ng, S. Turner, "A Complete Single-Chip Implementation of the JPEG Image Compression Standard," IEEE Custom Integrated Circuit Conference, pp. 12.2.1-12.2.4, 1991.

[5]. K. Ogawa, T. Urano, K. Kondo, N. Mori, S. Morial, H. Yamamoto, S. Kato, "A Single Chip Compression/Decompression LSI Based on JPEG," IEEE Transactions on Consumer Electronics, Vol. 38, No. 3, Aug. 1992.

[6]. D. A. Luthi, P. Tong, P. A. Ruetz, "A Video-Rate JPEG Chip Set," IEEE Custom Integrated Circuit Conference, pp. 26.2.1-26.2.4, 1992.

[7]. M. Kovac, N. Ranganathan, "JAUGUAR: A Fully Pipelined VLSI Architecture for JPEG Image Compression Standard," Proceeding of the IEEE, Vol. 83, No. 2, Feb. 1995.

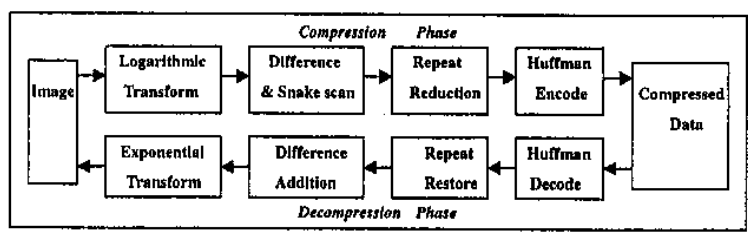

Fig. 1, Log-Exp Compression System

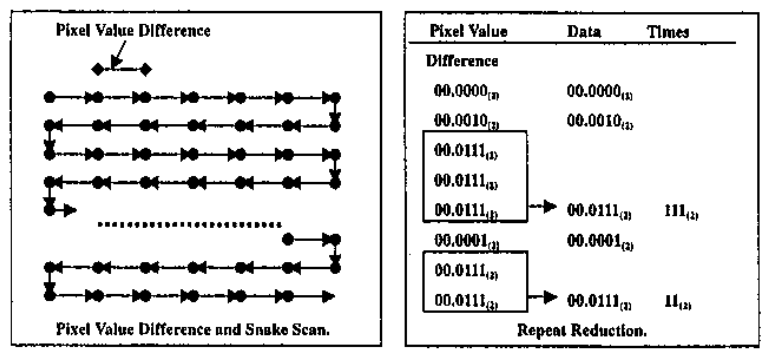

Fig. 2. Log-Exp Algorthm Deslgn.

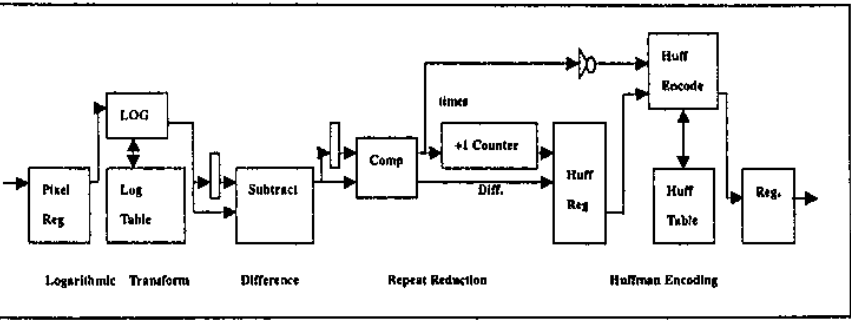

Fig. 3. LOG.EXP Encoder Architecture Diagram

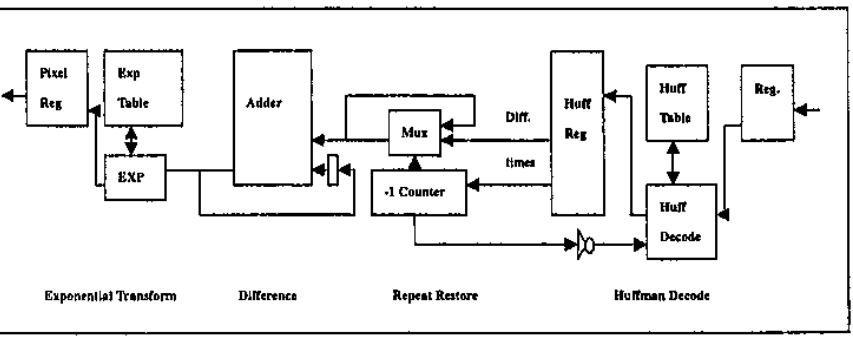

Fig. 4. LOG-EXP Decoder Architecturo Diegrem.

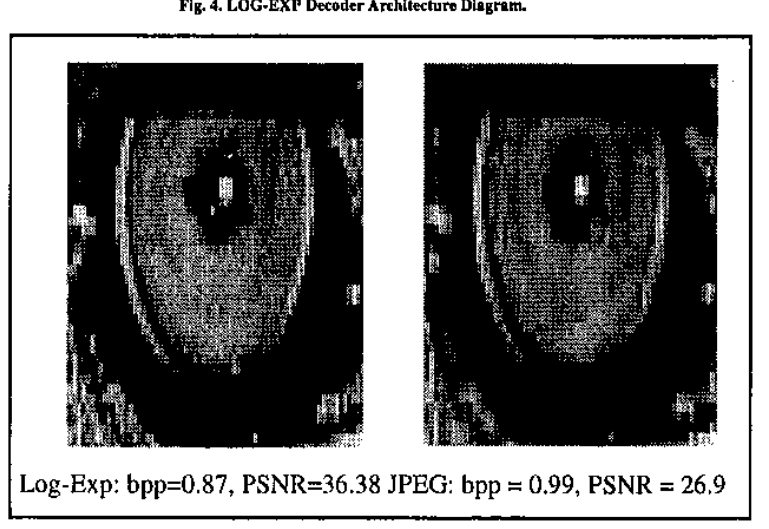

Fig. 5. The Zoom View Comparison for the left eye of the benchmark Image baboon. 